\title{
The Fabricare system: a multi-agent-based scheduling prototype
}

\author{
PAULO SOUSA, CARLOS RAMOS and JOSÉ NEVES
}

Keywords Holonic manufacturing systems, multi-agent systems, scheduling

\begin{abstract}
Several trends in society in general, and manufacturing in particular, have changed the way business was made in the last decades of the 20th century, setting new requirements for companies and individuals. The research question being addressed in this paper is concerned with the ability to build computer-supported manufacturing systems able to cope with current and future requirements. For this matter, a hypothesis based on the holonic and multi-agent paradigms is proposed. The paper describes a holonic architecture for manufacturing enterprises and a prototype system (named Fabricare) for manufacturing orders scheduling based on that architecture.
\end{abstract}

A negotiation mechanism called 'contract net with constraint propagation protocol' was developed for regulating the interaction between holons in the system. This protocol also implements a negotiation-driven scheduling procedure.

\section{Introduction}

Society has experienced evolving mutations since the dawn of ages, especially in the last half of the 20th century. In recent years several trends were observed in manufacturing and society, namely: market globalization; increasing product/services customization; increasing technology

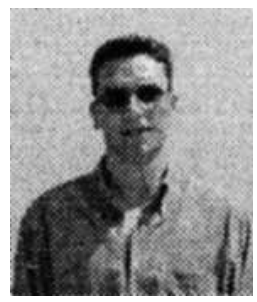

Authors: Paulo Sousa and Carlos Ramos, Instituto Superior de Engenharia do Porto, Rua Dr. António Bernardino de Almeida, 431, 4200-072 Porto, Portugal, E-mails: \{psousa, csr\}@dei. isep.ipp.pt. José Neves, Universidade do Minho, Largo do Paço, 4719 BRAGA Codex, Portugal, E-mail: jneves@di.uminho.pt.

Paulo Sousa studied computer science at Instituto Politécnico do Porto (Politechnic Institute of Porto Portugal - ISEP-IPP from 1994 to 1995 with a specialization in Industrial Informatics. He worked for three years as an application developer for a Portuguese software house in the field of electronic archive, database retrievals and component building. In 1996 became an assistant professor at ISEP-IPP. In 1998, he successfully concluded a post-graduation on 'Distribution Systems, Computer Architectures and Computer Communications' at Universidade do Minho (University of Minho, Portugal), and in 2002 achieved his PhD degree in Computer Science at Universidade do Minho with his work on Holonic Manufacturing Systems. He is currently an active member of the ISEP's Research Group on Knowledge Engineering and Decision Support (GECAD, http://www.gecad.isep.ipp.ptl/) and the technical contact point for the ISEP-IPP node of AgentLink - European Network of Excellence in Agent-Ifased computing. His main research interests are Computer Graphics, Distributed Intelligent Systems and Enterprise Application Architectures. More information can be found at http://www.dei.isep.ipp.pt/ psousa.

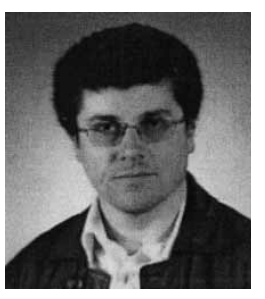

Carlos Ram was born in São Paulo, Brazil, in 1963. He got his BSc (licenciatura) degree in Electrical Engineering in 1986, and a PhD degree in Electrical and Computer Engineering in 1993, both from the University of Porto, Portugal. Currently he is Co-ordinator Professor at the Institute of Engineering-Polytechnic of Porto (ISEP-IPP). He is Director of the ISEP Research Group on Knowledge Engineering and Decision Support (GECAD, http://www.gecad.isep.ipp.pt/). He was responsible for $14 \mathrm{R} \& \mathrm{D}$ projects, and supervised $16 \mathrm{MSc}$ thesis and seven PhD works, and is supervising more on going $\mathrm{PhD}$ work. He has published more than 274 scientific papers. His main areas of interest are Artificial Intelligence and Manufacturing Systems, namely involving Decision Support, Knowledge-based Systems, and Multi-agent Technologies. 


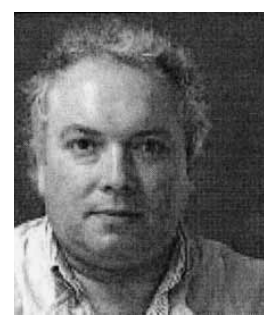

José Neves was born in Santa Cristina de Malta-Vila do Conde, Portugal, in 1948. He got his BSc (licenciatura degree in Chemical Engineering in 1976, at the University of Coimbra, Portugal, MSc and PhD degrees in Computer Science in 1981 and 1983, both from the University of HeriotWatt, in Edinburgh, Scotland. Currently he is Full Professor at the University of Minho (UM), in Portugal. He is responsible for the UM Research Group on Artificial Intelligence and co-ordinates several R\&D projects involving Artificial Intelligence basic research and application. He applied with success several Artificial Intelligence technologies like Logic Programming, Neural Networks, Constraints, Evolutionary - Programming, Multi Agent Systems, Negotiation and Argumentation in areas like Medicine, Law and Legal Domain, Electronic Commerce, Manufacturing, etc. He has had more than 150 scientific papers pubslished.

complexity; increasing number of competitors; decreasing product life cycles; and increasing quality requisites (Kusiak 1990, Solberg and Kashyap 1993, NGM 1997). Stability, certainty and predictability are giving place to change, uncertainty and unpredictability (Kidd 1994). In addition, a shift towards flexible manufacturing and customized products is evident (Atkinson and Court 1998).

From a technological point of view, it was observed that current manufacturing systems (e.g. computer-integrated manufacturing architectures) pose several drawbacks, namely excessive rigidity and centralization; high implementation costs; and inflexibility (Höpf 1994, Ueda 1994, Bongaerts et al. 1995, Parunak 1996, Tharumarajah et al. 1996, Gou and Luh 1997, Bussmann 1998, Kadar et al. 1998, Shen and Norrie 1999). Furthermore, it is expected that in the future manufacturing will be characterized by: globally distributed resources; small quantities and high variety of products; providing individual solutions tailored to each customer's specific needs; concurrent execution of all the activities in the manufacturing process (NGM 1997, CVM 1999).

In order to deal with the identified problems with current manufacturing systems and prepare them for the expected future scenarios, the new generation of manufacturing systems must possess such attributes as decentralization, distribution, autonomy, adaptability and incomplete information handling (Sousa et al. 2000b, c).

New approaches have been proposed, providing systems that are more adequate in a current and future social-economic context. Multi-agent systems (MAS) (Parunak 1998) and holonic manufacturing systems (HMS) (Valckenaers et al. 1994a, b, 1997) appear as promising approaches to this point.

This paper presents a system, named 'Fabricare', based on holonic principles, for dynamic scheduling of manufacturing orders. The system tries to achieve the following user's needs:

- High flexibility - cope with change in system's structure and behaviours (e.g. cardinality of resources, resource's functionality);

- High adaptability - cope with change in system's dynamics (e.g. machine breakdown) and in markets;
- High availability - reduce downtime (hence cost) and single point of failure.

These user needs were translated into objectives, which are seen in this work from a software point of view. That is, for instance, availability relates to the software system availability and not to machine or tool availability.

\section{System architecture}

This work tries to present an integrated view of a manufacturing system, based on the distributed manufacturing system paradigm, able to accomplish the requirements for the post-20th century society (Sousa et al. 2000a). In order to overcome these challenges, a solution based on holonic manufacturing systems has been proposed using multi-agent systems and extended logicprogramming. Although overlapping, the two concepts, MAS and HMS, complement each other, especially by using MAS as an implementation tool for HMS (Bongaerts 1998, Bussmann 1998, Kirsch et al. 1998, Sousa et al. 2000c, Ulieru et al. 2000). To the scope of this work, a holon is understood as a logical design entity in the system architecture, and is implemented as an agent using extended logic programming. Although not necessarily a matter of consensus, both words (holon and agent) can be used as synonyms in this paper.

In this solution, Fabricare, which is the Latin word for 'to manufacture', the main entities in the manufacturing process are modelled as holons, each one contributing with a small parcel of the overall system's functionality.

The Fabricare project uses a holonic architecture (figure 1) where several key functions of the manufacturing process are identified and modelled as holarchies (Sousa and Ramos 1998, 1999, Sousa 2000). These holarchies are composed of 'basic' holons such as product, task and resource, each one representing a core entity in the manufacturing system.

The core holons are grouped together in holarchies representing the major function of the manufacturing system. The design holon (DH) aggregates product holons $(\mathrm{PH})$, customer holons $(\mathrm{CH})$ and supplier holons $(\mathrm{SuH})$ 


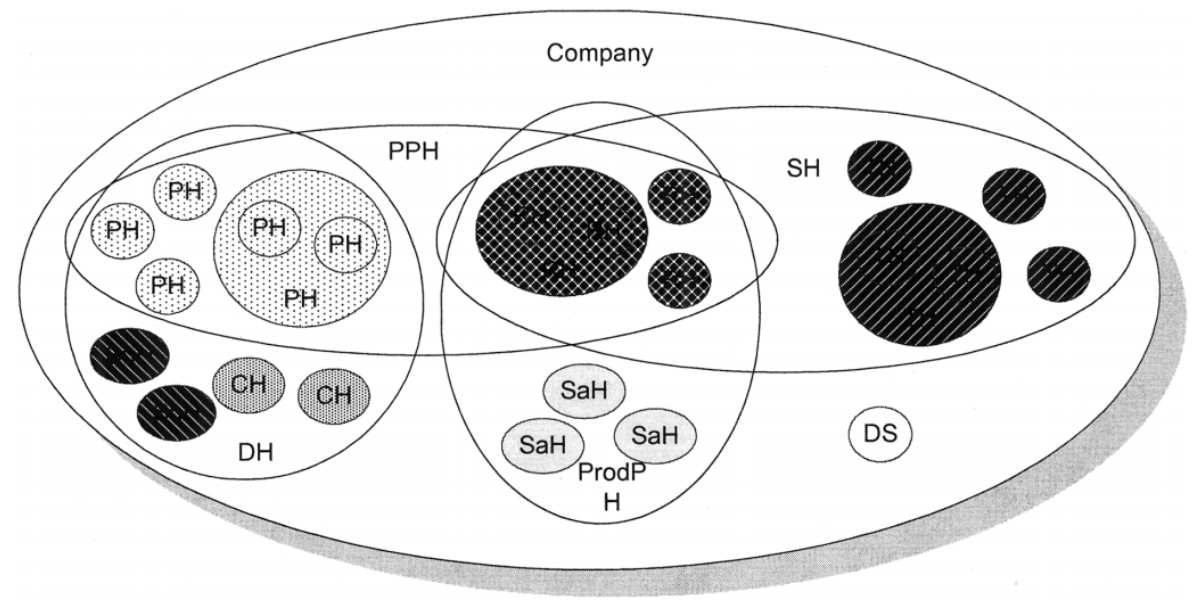

Figure 1. The architecture of the Fabricare project.

for the design activity. The process planning holon (PPH) aggregates resource holons $(\mathrm{RH})$ and product holons $(\mathrm{PH})$. The production planning holon (ProdPH) aggregates resource holons and sales holons $(\mathrm{SaH})$ to execute material requirements planning and capacity planning. The scheduling-holon ( $\mathrm{SH}$ ) aggregates resource holons and task holons $(\mathrm{TH})$ to dynamically schedule manufacturing orders. Additionally, there is the directory service (DS) that acts a repository for the system providing information about running holons.

\section{Holon interaction}

In order to regulate the interaction between Fabricare's holons, a protocol is used, aiming to achieve cooperation. The participants in this protocol are task holons and resource holons. Task holons represent a manufacturing order to be executed by the shopfloor, while resource holons represent the physical machinery on the shopfloor.

For the scheduling of task's sub-operations, the task holon will negotiate with resource holons, using an extension of the contract net protocol (Smith 1980, Davis and Smith 1983) with a cooperation phase between service providers (i.e. resource holons). The resource holons will use constraint propagation in order to guarantee the relationships among different operations that aim at the same task. This new protocol is called 'contract net with constraint propagation protocol' (CNCPP) (Sousa and Ramos 1997, 1998, 1999, Sousa et al. 1999, Sousa 2000) (figure 2).

After receiving a request, each resource holon will be 'forward-influenced' by its predecessors and will forwardinfluence its successors. Likewise, each resource holon will be backward-influenced before making its bid, and will backward-influence its predecessors (Sousa and Ramos
1999, Sousa 2000). The 'forward' and backward-influence operations consist in adjusting the beginning and the end of each resource's agenda of free time intervals with the agenda of predecessor/successor resources (i.e. delete from the list of free time intervals, those intervals where it is impossible to schedule the operation because the part is still being processed by other resources (Ramos et al. 1995)). The set of resources participating in a specific negotiation is built at the beginning of that negotiation by the task holon. If new resource holons are added to the system they will not be considered for running negotiations but will enter future ones.

Since multiple tasks can be negotiated at the same time, conflicts may arise if some resources are used in the same time interval for different tasks. In these scenarios, resource holons have an indecision problem (Sousa and Ramos 1998) since they cannot guarantee the delivery of both tasks. In order to overcome this problem, a solution is proposed which involves a pre-negotiation step in the protocol. Before beginning negotiation, each task holon will ask for authorization from the scheduling holon, which maintains a list of negotiating resources and respective time windows. Only in the case of non-overlapping a 'green light' will be given to the negotiation.

The system is prepared for overlapping functionality on the resource holons, i.e. different resource holons can perform the same operation (e.g. drill). A task holon will receive the production plan for a product with indication of the necessary operations, and will request them to every resource holon able to perform each operation. This causes a combinatorial explosion in the number of exchanged messages between agents (with an $O\left(r^{n}\right)$ complexity, where $r$ is the number of resources and $n$ the number of operations). To decrease the number of messages a modification was made in the protocol, in 

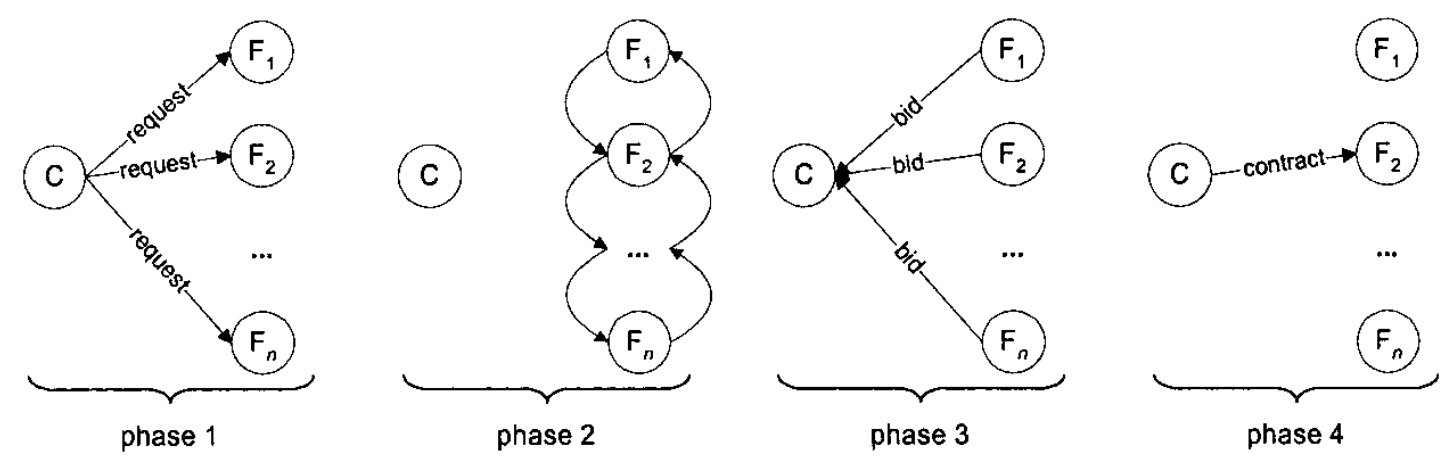

Figure 2. Contract net with constraint propagation protocol (CNCPP).

which combinations from predecessor resources are clustered before sending them to successor resources (Sousa 2000). This change has made the complexity of the problem $O(n)$ in which respects the number of exchanged messages. However, the problem of a combinatorial explosion in the search space still exists (possible solutions for this problem are being addressed).

The scheduling procedure is adapted from a centralized method described in Ramos et al. (1995) and Almeida (1995), based on agendas, behaviours and due dates. Behaviours are static resource allocation mappings, which are pre-generated for each production plan. The calculations for time intervals are performed by the resource holons when executing the contract net with constraint propagation protocol. When compared to the original scheduling procedure from Ramos et al. (1995), Fabricare's approach has the ability to use alternative resources for each operation as well as using the same resource for more than one operation in the same production plan.

The same protocol can be used for renegotiating the task in case of machine breakdowns or other unexpected events (Sousa and Ramos 1999). In that case, the task holon will be informed by the resource holon where the event originated, and will begin negotiations for the operation(s) previously contracted to that resource with other resource holons following the same steps previously described. At this time, if rescheduling is not possible, the task will be completely abandoned, and a manual scheduling of that task must be engaged.

\section{Operational description of main holons}

The key entities participating in the scheduling process (activity chosen as the test case) are the physical resources and the manufacturing orders. These two entities are represented in the system by resource holons and task holons respectively. This section details the internal behaviour of each one of these holons.

\subsection{Task holon}

A task holon represents a manufacturing order to execute a certain quantity of a specific product on the shop floor. This kind of holon has as its objective to schedule the order and monitor its execution.

Its life cycle (figure 3 ) begins when the manufacturing order is created (either to fulfil a customer order or to balance stocks). During its existence the task holon will negotiate with resource holons the execution of the operations needed to perform the ordered product. The holon will cease existing when the order is fulfilled or cancelled.

After obtaining information about the order, the task holon negotiates with resource holons using CNCPP. The holon will then wait for the bids and evaluate them, in order to select one (if possible). If it is not possible to schedule the order, the task holon will recombine the resources and perform a new negotiation. A renegotiation may also be necessary if the order's condition changes e.g. anticipated due date, delayed, etc. (Sousa and Ramos 1997, 1999). The evaluation of bids is performed taking into account a prioritized list of criteria. The following criteria have been implemented in the prototype: (i) first valid solution; (ii) least costly solution; and (iii) greatest slack till due date. The cost of a solution is determined by the cost of performing the specified operations in a specific resource.

\subsection{Resource holon}

A resource holon represents the current state of a physical resource on the shopfloor. The resource's list of activities is called agenda, stating what to do and when. The resource is able to perform operations 
necessary to execute products (e.g. drill). A resource holon can represent a single resource or a work cell composed of several resources.

The objective of a resource holon is to control the physical equipment, provide information about its abilities and status to the system and manage the scheduled activities. Its life cycle is very long, since it is

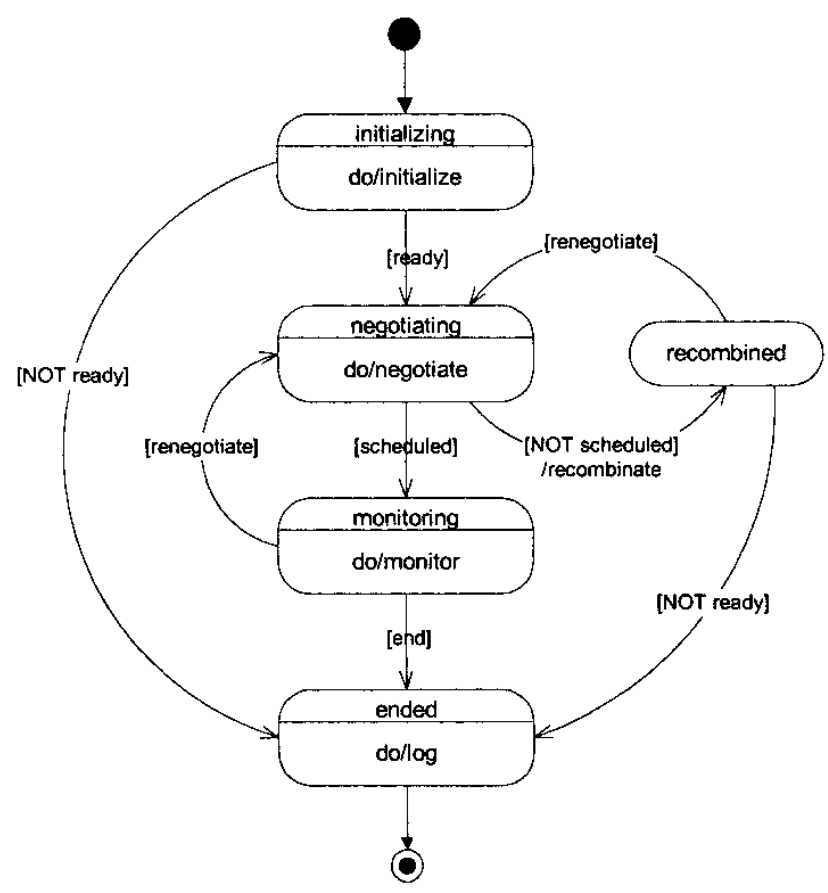

Figure 3. Task holon's life cycle. expected that a resource is fully operational for long periods of time. During its existence, the resource holon executes the commands sent by the resource controller and negotiates with task holons the scheduling of manufacturing orders.

During initialization, the holon builds its initial agenda, registers in the directory service and holarchies to which it belongs (e.g. scheduling, process planning). The negotiation process of the holon is guided by the execution of the CNCPP state machine (figure 4) for each conversation currently taking place with task holons. Upon reception of a service request (for the possible execution of one or more operations) the resource holon will check its availability and engage in negotiation with other resource holons for propagating constraints between the operations. After calculating its feasible intervals for each request, it will send a bid and wait for the task holon's reply (accept or decline). There is a cost mechanism associated with operations and resources, such that a resource holon replies to a task holon with a bid specifying the price (in abstract units) of performing that operation.

\section{The prototype suite}

\subsection{Prototype description}

Figure 5 presents the Fabricare scheduling prototype suite, composed of several applications. The 'configuration

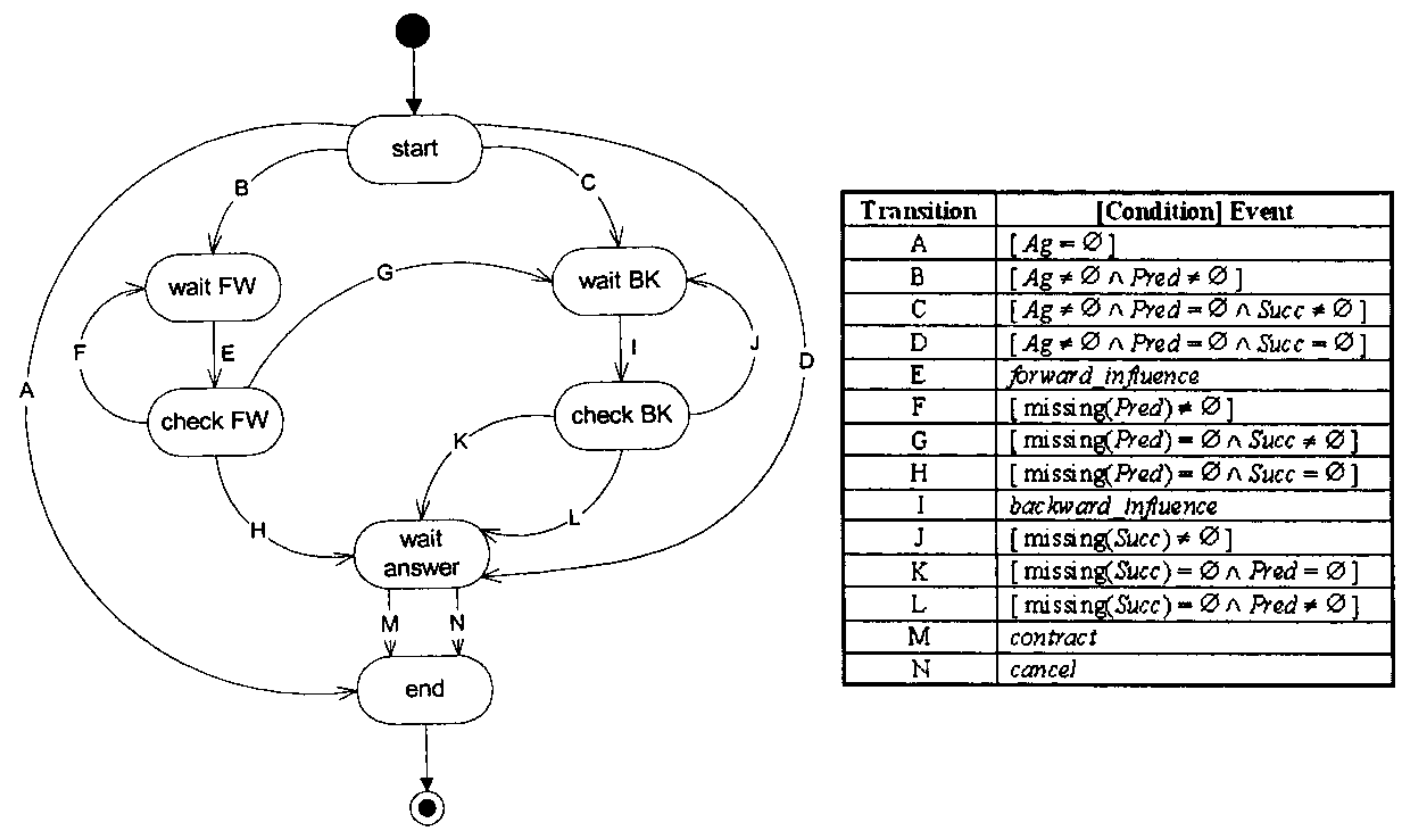

Figure 4. State diagram for one negotiation. 


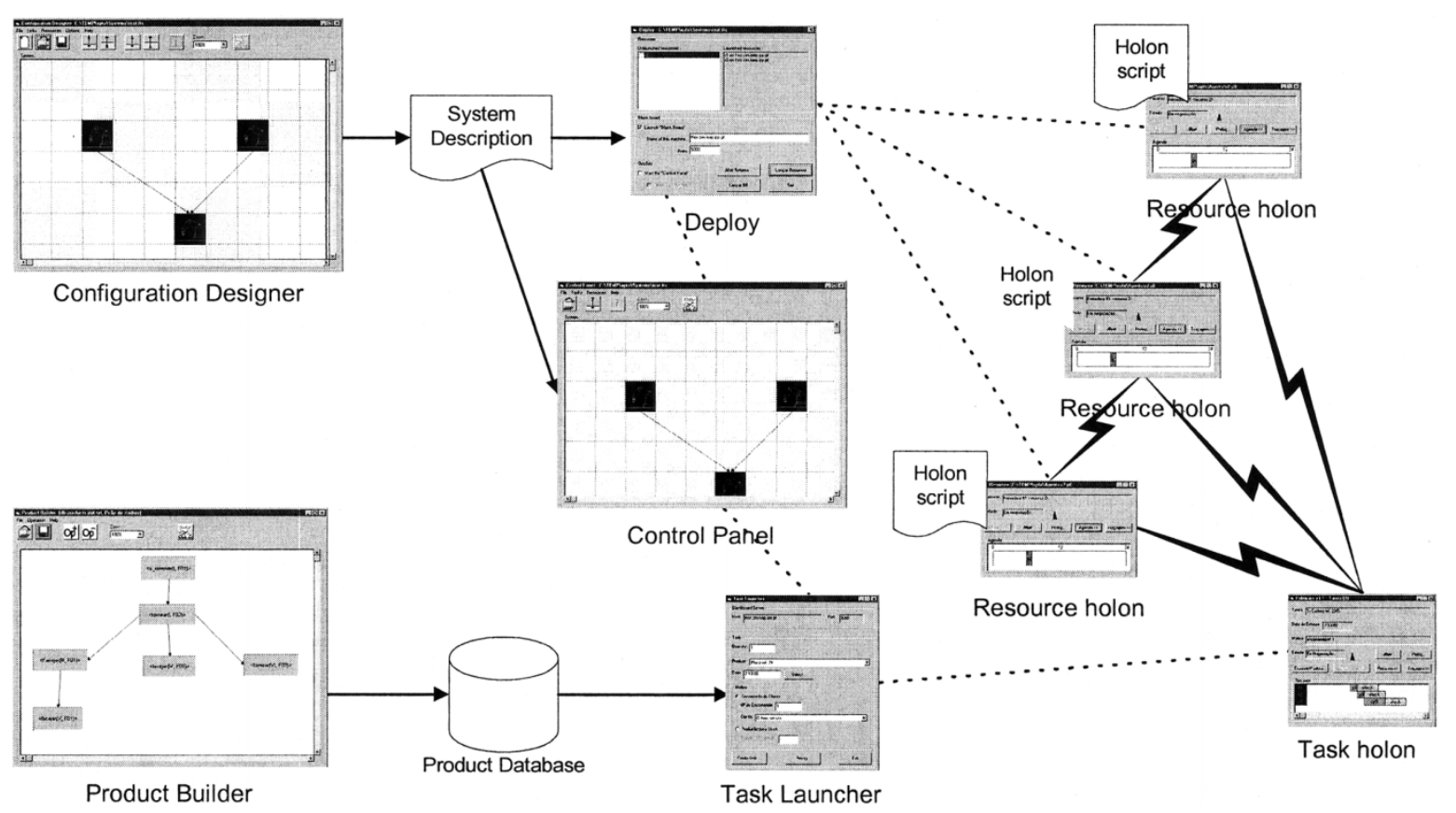

Figure 5. Fabricare's scheduling application suite.

designer' allows specifying the resource agents in the factory plant and, to some extent, represent graphically the physical layout of the resources. The system description is read by the 'deployment' tool, which launches 'resource holons' on the desired machines. Each resource holon is composed of a kernel and individual characteristics and behaviours specified in the holon script written in Prolog representing the 'mental' state of the holon (e.g. resource's agenda), as well as specific clauses for the Fabricare holon kernel (e.g. name). The 'control panel' is the interface to the system's operation, monitoring and controlling running holons. This tool also allows the user to launch tasks (manufacturing orders) in the system by evoking the 'task launcher' tool, which prompts the user for data about the order and dynamically creates a 'task holon' for that order. One last tool in the suite is the 'product builder', which allows to generate graphically a product's process plan. The several operations in the plan are the abilities of the physical resources (modelled in the resource holons).

The system is very dynamic in what concerns its holons, i.e. resource holons depend on the system description file; task holons depend on the existing tasks (dynamic events). Each negotiation uses the set of holons that are present and available at that time, thus giving the system a high degree of adaptability to the dynamic nature of the manufacturing arena (e.g. resource in maintenance or overloaded).

The holons are extended logic programs written in Prolog with the ability to handle negative and incomplete knowledge (Sousa et al. 2000b). The decision procedure is not yet totally driven by this kind of knowledge; however, real-life scenarios where only partial information is available have been identified (Sousa 2000, Sousa et al. 2000b) and modelled (e.g. resource holons will use this information to generate low commitment schedules into their agendas).

\subsection{Experiments}

This section presents some of the experiments made with Fabricare essentially in which concerns the scheduling procedure (comparing Fabricare's results with the original results from Almeida (1995)). A more comprehensive set of experiments can be found in Sousa (2000).

Almeida (1995) presents a scenario with tree tasks (following the plans in figure 6) for the production of two items of each product. The initial conditions of the problem are described in table 1 .

Figure $7 \mathrm{a}$ shows the results obtained by Almeida (1995) and figure 7b shows the results obtained by Fabricare using the 'first valid solution' selection criteria. The new scheduling procedure allows for the use of new time intervals since it does not use a static mapping (behaviours according to Almeida (1995) and Ramos et al. (1995)), and generates the feasible intervals by considering buffers between the resources. 


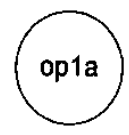

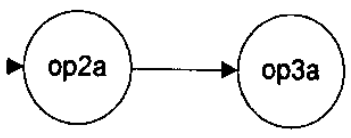

(a)
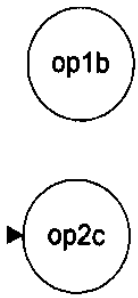

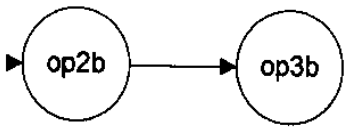

(b)

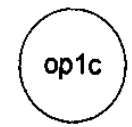

(c)

Figure 6. Production plans for test scenario 1.

Table 1 . Test conditions for test scenario 1 .

\begin{tabular}{lccl}
\hline Resource & Operation & Duration & Initial agenda \\
\hline Ml & opla & 2 & {$[(5,7),(11,13),(18,20)]$} \\
& op3b & 1 & \\
M2 & oplc & 2 & \\
& op2a & 3 & {$[(8,10),(15,18)]$} \\
M3 & op1b & 2 & \\
& op3a & 1 & {$[(1,5),(16,19)]$} \\
& op2b & 1 & \\
\hline
\end{tabular}

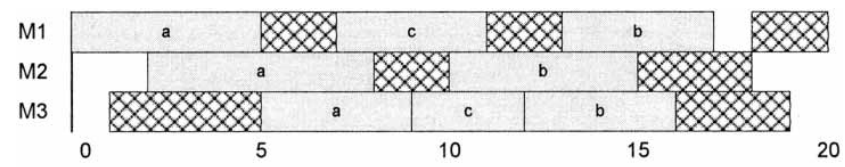

(a)

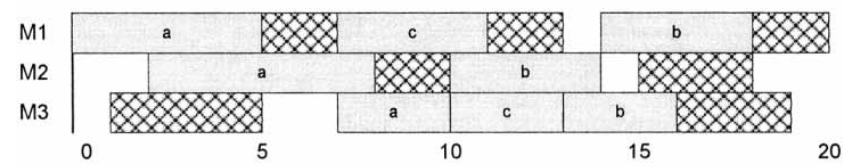

(b)

Figure 7. Results of test scenario 1.

Another experiment was made considering a task with a sequential production plan (op1 op2 op3) for the production of two items. The initial agenda for each resource as well as the cost associated with each operation for the several resources are presented in table 2.

This experiment considers the existence of alternative resources for the realization of opl (R1 and R4) as well as for the realization of op2 (R2 and R5), hence generating four alternative solutions shown in table 3. Furthermore, the cost of executing an operation varies according to the resource it is performed in.

The selection criterion used was 'least costly solution', which eliminated the first two proposals. The other two proposals have identical costs (and identical schedules since R5 had no initial commitments), thus the task holon used the 'first valid solution' criterion as secondary selection criterion, resulting in the following assignment (including slack): $\mathrm{R} 4 /(6,10), \mathrm{R} 2 /(7,21)$ and $\mathrm{R} 3 /(10,24)$.

For relatively simple production plans (maximum five operations) with some alternatives (maximum eight alternative plans) the performance obtained was quite good since solutions were given (on average) in less than 1.10 seconds. The test bench consisted of a network of Microsoft Windows workstations and the results were measured from the control panel component and include network latency and communications overhead - thus this is the system response time from the user point of view. These results allow expecting a convergence of solution considering soft real-time constraints. Obviously, more complex production plans and several alternative resources may produce an exponential explosion of the solution space and resulting in the degradation of the system performance.

\section{Conclusions and future work}

This paper presented a system based on holonic concepts and a prototype implementation of that system that may be characterized by:

- The main entities in the manufacturing systems are represented as holons while the core functions are represented as holarchies.

- It is a distributed application based on the multiagent and holonic paradigms.

- The implementation of task holons and resource holons (as well as other support holons) for the dynamic scheduling of manufacturing orders.

- The knowledge and control of each agent is separated through holon scripts.

- It is open and flexible in which concerns the number of holons.

- The holons have the ability to represent and handle incomplete information in their knowledge bases. 
Table 2. Test conditions for scenario 2.

\begin{tabular}{lcccl}
\hline Resource & Operation & Duration & Cost & $\begin{array}{l}\text { Initial } \\
\text { agenda }\end{array}$ \\
\hline R1 & opl & 1 & 100 & {$[(1,2)]$} \\
R2 & op2 & 1 & 100 & {$[(6,7)]$} \\
R3 & op3 & 3 & 300 & {$[(7,10)]$} \\
R4 & op1 & 1 & 90 & $\begin{array}{l}{[(1,2),(3,6),} \\
(10,12),\end{array}$ \\
& & & & $(17,18)]$ \\
R5 & op2 & 1 & 100 & {[]} \\
\hline
\end{tabular}

Table 3. Results of test scenario 2.

\begin{tabular}{|c|c|c|c|c|}
\hline Solution & Cost & Resource & $\begin{array}{l}\text { Proposed } \\
\text { intervals }\end{array}$ & $\begin{array}{c}\text { Partial } \\
\text { cost }\end{array}$ \\
\hline \multirow[t]{3}{*}{ R1-R5-R3 } & 1,000 & R1 & {$[(2,20)]$} & 200 \\
\hline & & R5 & {$[(3,21)]$} & 200 \\
\hline & & R3 & $110,24)$ & 600 \\
\hline \multirow[t]{3}{*}{ R1-R2-R3 } & 1,000 & Rl & {$[(2,20)]$} & 200 \\
\hline & & $\mathrm{R} 2$ & {$[(7,21)]$} & 200 \\
\hline & & R3 & {$[(10,24)]$} & 600 \\
\hline \multirow[t]{3}{*}{ R4-R5-R3 } & 980 & $\mathrm{R} 4$ & $\begin{array}{c}{[(6,10),(12,17),} \\
(18,20)]\end{array}$ & 180 \\
\hline & & R5 & {$[(7,21)]$} & 200 \\
\hline & & R3 & {$[(10,24)]$} & 600 \\
\hline \multirow[t]{3}{*}{ R4-R2-R3 } & 980 & $\mathrm{R} 4$ & $\begin{array}{c}{[(6,10),(12,17),} \\
(18,20)]\end{array}$ & 180 \\
\hline & & $\mathrm{R} 2$ & {$[(7,21)]$} & 200 \\
\hline & & R3 & {$[(10,24)]$} & 600 \\
\hline
\end{tabular}

This system resembles the distributed nature of manufacturing, thus allowing for a better modelling of the real system. While other co operative communities operate with agents representing resources or systems, the Fabricare system combines resource-based holons with task-based holons. The main advantage is the easy access to task activities that are supported in task-based holons as well as high adaptability to the dynamic nature of resource conditions and availability.

The negotiation protocol presented to regulate the interaction among the several agents in the system contract net with constraint propagation protocol (CNCPP), may be characterized by:

- Explicit cooperation phase between service providers (i.e. resources) motivated by the need to coordinate temporal relations of a task's operations.

- Dynamic participants, using the information of running agents stored in the directory service.

- Conflict avoidance by serializing overlapping negotiation (concurrent time-windows for different resources/tasks).

- The existence of alternative resources for the same operation may cause combinatorial explosion of the solution space.
- The number of exchanged messages is of the order $O(n)$.

- A renegotiation phase can be triggered by abnormal events (e.g. machine breakdown).

The main advantage of this protocol is the existence of explicit cooperation between resource agents in order to guarantee feasible schedules satisfying the precedence relations between operations in the same task. One of the major issues for future work is the combinatorial explosion of the number of generated solutions. A possible solution for this problem may be to guide the selection process of the task holon by applying meta-rules based on the complexity of the problem and the available time. This has the side effect of introducing soft real-time constraints in the protocol to ensure at least one solution in useful time.

The scheduling procedure (intimately related to the protocol just described) was adapted from a proven centralized method, but introduced the following new characteristics:

- Use of a distributed approach with several computational processes (resource holons and task holons) performing partial calculations and having partial problem data.

- Use of cost by operation by resource which allows a new parameter to obtain different solutions.

- Possibility of several resources for the same operation generating several alternative schedules considering resource's agendas and costs.

- Use of buffers between resources allowing for greater flexibility than with the static behaviour model.

The main advantage of this scheduling procedure is the fact that it allows the use of several resources for the sane operation and allows for greater flexibility by not using behaviours as in the original method.

Thus, it is possible to say that the Fabricare system achieves the objectives stated in section 1 in the following way:

- High flexibility - since the structure of the system is 'open and flexible' the number of resources may vary, with resource holons being added or removed on the fly. Furthermore, additional functionality can be introduced at each resource. Task holons use a dynamic query to find out which resources to negotiate with, thus getting a snapshot of the system at that specific time instead of relying on a static a priori mapping of the world.

- High adaptability - since there is some support to a renegotiation phase in the CNCPP, the system may adapt to abnormal events. However, there is still some work to be done in this matter: for instance, 
introduce some notion of priority or budget to the task that will allow it to 'persuade' other scheduled tasks to give up resource time in order to fulfil its schedule.

- High availability - since there is no central control element in the system, single points of failure do not exist which makes the system more robust to workstation or network breakdown. Additionally, having duplicated functionality in different resources allows the system to continue operating using the remaining pool of resources.

The problem being addressed here is related to the ability to build and maintain computer-supported manufacturing systems able to cope with recent (and expected future) requirements, giving the socialeconomic context of the new digitized, customized, global society. In a way, the answer to this question can be given by organizing the manufacturing system into 'small' units structured according to the holonic theory.

Essentially, it is argued that in order to overcome the rising customization of goods and services as well as other trends in society and economy, it is necessary to build systems with such characteristics as: distribution, decentralization, autonomy, dynamism, reactivity, flexibility, adaptability and agility. Although current manufacturing systems do not present them, this kind of properties can be found in holonic systems. These were the principles and goals guiding the development of the Fabricare system.

\section{Acknowledgments}

The authors would like to thank FCT-'Fundação para a Ciência e Tecnologia' (Portuguese Foundation for Science and Technology) and programmes POCTI and FEDER for their support in R\&D projects. Acknowledgements are also given to the Scientific Council of ISEP/IPP and to FLAD - Fundação LusoAmericana para o Desenvolvimento' (PortugueseAmerican Foundation for Development). Additionally, we acknowledge the European Commission which supported some ESPRIT and IST programmes in which we were involved.

\section{References}

AlmeidA, A., 1995, Escalonamento dinåmico de tarefas industriais sujeitas a prazos de entrega. Dissertação de Mestrado. Faculdade de Engenharia da Universidade do Porto. (MSc thesis in Portuguese).
Atkinson, R., and Court, R., 1998, The New Economy Index: Understanding America's Economic Transformation (Washington, DC: Progressive Policy Institute).

BongaERTS, L., 1998, Integration of scheduling and control in holonic manufacturing systems. PhD thesis, Production and Automation Division, Katholieke Universiteit Leuven. LEUVEN, Belgium.

Bongaerts, L., Valckenaers, P., Van Brussel, H., and Wyns, J., 1995, Schedule execution for a holonic shop floor control system. ASI'95, Proceedings of the First Advanced Summer Institute.

Bussmann, S., 1998, An agent-oriented architecture for holonic manufacturing control. IMSEurope'98, Proceedings of the First International Workshop on Intelligent Manufacturing systems, pp. 1-12.

CVM 1999, Visionary Manufacturing Challenges for 2020, (Washington, DC: National Academic Press).

DAVIS, R., and Smith, R., 1983, Negotiation as a metaphor for distributed problem solving Artificial Intelligence, $\mathbf{2 0}(1)$, 63-109.

Gou, L., and LuH, P., 1997, holonic manufacturing scheduling: architecture, Cooperation mechanism, and implementation. Proceedings of IEEE/IASME International Conference on Advanced Intelligent Mechatronics.

HöPf, M., 1994, Holonic manufacturing sytems-the basic concept and a report of IMS test Case 5. In J. Knudesen et al. (eds.) Sharing CIMSolutions (IOS Press).

Kádár, B., Monostori, L., and Szelke, S., 1998, An objectoriented famework for developing distributed manufacturing-architecture. Fournal of Intelligent Manufacturing, 9(2), 73-179.

Kelly, K., 1998, New Rules for the New Economy: 10 Radical Strategies for a Connected World (Harmondsworth: Penguin).

KIDD, P., 1994, Agile Manufacturing, Forging New Frontiers Reading, MA: Addison-Wesley).

Kirsch, D., Wieczorek, D., and Albayrak, S., 1998, Open agent architecture for the realization of holonic manufacturing systems. IMS-Europe'98, Proceedings of the First International Workshop on Intelligent Manufacturing Systems, pp. $1-12$.

KusiaK, A., 1990, Intelligent Manufacturing Systems (Englewood Cliffs, NJ Prentice-Hall).

NGM, 1997, Next Generation Manufacturing - Framework for Action. Next Generation Project Report, Agility Forum.

PARUnAK, H., 1996, Applications of distributed artificial intelligence in industry. In O'Hare and Jennings (eds.) Foundations of Distributed Artificial Intelligence (Chichester: Wiley Interscience).

PARUnAK, H., 1998, What can agents do in industry and why? CIA '98, Proceedings of the Second International Conference on Co-operative Information Agents.

Ramos, G., Almetda, A., and VAle, Z., 1995, Scheduling manufacturing tasks considering due dates: a new method based on behaviours and agendas. Proceedings of the International Conference on Industrial and Engineering Applications of Artificial Intelligence and Expert Systems, pp. 745-751.

Schonfeld, E., 1998, The customized, digitized, have-it-yourway economy. Fortune, 28 September.

Shen, W., and Norrie, D., 1999, Agent-based-systems for intelligent manufacturing: a state-of-the-art survey. Knowledge and Information Systems 1(2), 129-156.

SMITH, R., 1980, The contract net protocol. IEEE Transactions on Computers, $\mathbf{C}-29$ (12). 
SolberG, J., and KASHYAP, R., 1993, ERG Research in intelligent manufacturing systems. Proceedings of the IEEE, 81(1), 25-41.

Sousa, P., 2000, agentes inteligentes em sistemas holónicos de produção. Tese de Doutoramento, Universidade do Minho. Braga, Portugal ( $\mathrm{PhD}$ thesis in Portuguese).

Sousa, P., and Ramos, C., 1997, Proposal of a scheduling holon for manufacturing. PAAM'97, Proceedings of the Second International Conference on the Practical Application of Agents and Multi-agents Technologies, pp. 55-268.

Sousa, P., and Ramos, C., 1998, A dynamic scheduling holon for manufacturing order. Fournal of Intelligent Manufacturing, 9(2), 107-112.

Sousa, P., and Ramos, C., 1999, A distributed architecture and negotiation protocol for scheduling in manufacturing systems, Computers in Industry, 38(2), 103-113.

Sousa, P., Ramos, C., and Neves, J., 1999, Contracting tasks between autonomous resources - an application to dynamic scheduling of manufacturing orders. PAAM'99, Proceedings of the Fourth International Conference on the Practical Application of Agents and Multi-agent Technologies, pp. 345-362.

Sousa, P., Ramos, C., and Neves, J., 2000a, Fabricare: an integrated view of a distributed manufacturing system. IAS-6, Proceedings of the sixth International Conference on Intelligent Autonomous Systems, pp. 423-428.

Sousa, P., Ramos, G., and Neves, J., 2000b, Manufacturing entities with incomplete information. Studies in Informatics and Control fournal, 9(2), 79-88.
Sousa, P., Silva, N., Heikkila, T., Kallingbaum, M., and VAlcknears, P., 2000c, Aspects of co-operation in distributed manufacturing systems. Studies in Informatics and Control Fournal, 9(2), 89-110.

Tharumarajah, A., Wells, A., and Nemes, L., 1996, Comparison of the bionic, fractal and holonic manufacturing concept. International Fournal of Computer Integrated Manufacturing, 9(3), 217-226.

UedA, K., 1994, Biological Manufacturing Systems (Tokyo: Kogyochosakai).

Ulieru, M., Stefanoid, D., and Norrie, D., 2000, Holonic reconfiguration of manufacturing production by fuzzy multi-agent modelling. IAS-6, Proceedings of the sixth International Conference on Intelligent Autonomous Systems, pp. 543-548.

Valckenears, P., Bonneville, F., Van Brussel, H., BONGAERTS, L., and WYNS, J., 1994a, Results of the holonic control system benchmark at KU LEUVEN, Proceedings of the International Conference on Computer Integrated Manufacturing and Automation Technology, pp. 128-133.

Valckenears, P., Van Brussel, H., Bongaerts, L., and Wyns, J., 1997, Holonic manufacturing systems. Fournal of Integrated Computer Aided Engineer, 4(3), pp. 191-201.

Valckenears, P., Van Brussel, H., Bonneville, F., Bongaerts, L., and Wyns, J., 1994b, IMS test case 5: holonic manufacturing systems. IMS Workshop at IFAC'94. 